\title{
PROKLA-Redaktion
}

\section{Editorial: Soziale Gerechtigkeit}

Zum Wahlsieg von Rot-Grün hatte 1998 nicht zuletzt die Wahrnehmung einer zunehmenden „sozialen Schieflage“ beigetragen, für welche die konservativ-liberale Regierung mitverantwortlich gemacht wurde. Nicht nur waren die Unternehmensgewinne und erst recht die Aktienkurse weit stärker gestiegen als die Einkommen der lohnabhängigen Bevölkerung, zusätzlich wurden gerade die hohen Einkommen durch Steuerreformen und neu geschaffene Abschreibungsmöglichkeiten überproportional entlastet. Die wachsende materielle Ungleichheit wurde nicht als unabänderliche Begleiterscheinung des Kapitalismus hingenommen, sie wurde vielmehr als „Gerechtigkeitslücke“ aufgefaßt, die dringend einer staatlichen Korrektur bedürfte. Wie die Bundestagswahl von 1998 zeigte, führte die offensichtliche Zunahme an materieller Ungleichheit auch in der individualisierten „Erlebnisgesellschaft“ noch zu erheblicher (wahl)politischer Mobilisierung. „Soziale Gerechtigkeit" - wie diffus auch immer verstanden - hatte sich als Grundwert erwiesen, der nicht derart offensichtlich und auf Dauer verletzt werden konnte, wie dies von der Regierung Kohl vorexerziert wurde. Nach wie vor spielte in der politischen Kultur der Bundesrepublik der Anspruch, der Staat möge für einen gewissen sozialen Ausgleich sorgen, statt die materiellen Ungleichheiten noch weiter zu verschärfen, eine entscheidende Rolle (vgl. dazu den Beitrag von Peter Lohauß).
Entgegen den in sie gesetzten Erwartungen knüpfte die rot-grüne Wirtschaftsund Finanzpolitik spätestens nach dem Rücktritt Oskar Lafontaines dann aber fast nahtlos an die neoliberalen Konzepte ihrer konservativen Vorgänger an (vgl. dazu die Beiträge in PROKLA 116, September 1999). Nun sind die geübten WahlbürgerInnen zwar längst daran gewöhnt, dass die Parteien nicht halten, was sie im Wahlkampf versprochen haben. Und es ist auch nichts Neues, dass gerade ehemalige Oppositionsparteien, sobald sie an die Regierung gekommen sind, ganz schnell von anscheinend unerbittlichen „Sachzwängen“ eingeholt werden - sprich den Interessen derjenigen Gruppen, deren Machtpositionen eben nicht zur Wahl stehen. Dass große Aufbrüche unrühmlich enden, sollte niemanden mehr überraschen. Während in der Vergangenheit aber eher still und leise von alten Versprechungen abgerückt wurde, konnte man in den letzten beiden Jahren insbesondere bei der deutschen Sozialdemokratie erleben, wie eine solche Kehrtwende offensiv als programmatische Erneuerung verkündet wurde (die englische Labour Party hatte eine ähnliche Wende als „New Labour“ immerhin schon vor ihrem Wahlsieg zum Programm erhoben.) Was sich bei der SPD mit dem 1999 veröffentlichten „Schröder-Blair"-Papier bereits angekündigt hatte, nahm in diesem Jahr mit der Debatte um ein neues Grundsatzpro- 
gramm bereits eine konkretere Gestalt an. Zwar rückt auch die SPD nicht von „Sozialer Gerechtigkeit“ als einem Grundwert $a b$, doch wird diese jetzt in einem ganz anderen Rahmen gesehen. Kernpunkt sozialer Gerechtigkeit ist für die SPD nicht mehr der Abbau von materiellen Ungleichheiten (bzw. die Linderung von deren Auswirkungen - an einen tatsächlichen Abbau ungleicher Besitzverhältnisse ist in einer kapitalistischen Gesellschaft, die diese Ungleichheit $\mathrm{zu}$ ihrer Grundlage hat, sowieso nicht zu denken); statt dessen wird jetzt die leistungssteigernde Wirkung von Ungleichheit hervorgehoben. Die vertrauten neoliberalen Floskeln - die sich die Mehrheit der grünen Wirtschafts- und FinanzpolitikerInnen schon vor einiger Zeit zu eigen gemacht hatten - tauchen nun, wenn auch nicht mehr ganz so laut und polternd wie bei den Konservativen der 80er Jahre, als brandneues Gedankengut einer „modernen“ SPD auf. Was durch Deregulierung und Globalisierung in den 80er und 90er Jahren schon praktisch vorangetrieben wurde, die Vermarktlichung aller gesellschaftlichen Verhältnisse und der Rückzug des Staates aus jeder steuernden oder korrigierenden Funktion, das wird jetzt auch von den politischen Kräften, die früher zumindest für ein Minimum an Widerstand gegen eine solche Entwicklung gut waren, offensiv legitimiert. Mit dieser „mentalen Kolonialisierung“ der SPD, die in ihrer längerfristigen Wirkung für das politische Klima in der Bundesrepublik kaum zu unterschätzen ist, setzt sich Birgit Mahnkopf in ihrem Beitrag auseinander.

Der Abbau staatlicher Verschuldung mit welchen sozialen Folgen auch immer erkauft - war stets einer der Kernpunkte liberaler Wirtschaftspolitik. Mit den Ver- trägen von Maastricht wurden den Mitgliedsländern der Eurozone sogar strikte nominelle Obergrenzen für die erlaubte Neuverschuldung vorgeschrieben. Der rot-grünen Bundesregierung genügt diese Begrenzung der Neuverschuldung aber nicht mehr. Finanzminister Eichel, der sich im Sparen von keinem Konservativen übertreffen lassen will, strebt $a b$ 2007 sogar Haushaltsüberschüsse an. Da die Einnahmen nicht beliebig wachsen werden, sind Ausgabenkürzungen programmiert und so wird von Eichel wie zuletzt bei einer Rede am 9. Oktober in der Humboldt-Universität Berlin schon mal vorsorglich gefordert, der Sozialstaat müsse sich „auf das Wesentliche beschränken“. Neu ist nicht diese Politik, sondern allenfalls ihre Begründung durch die Erfordernisse der „Generationengerechtigkeit“: Man wolle der nächsten Generation keine drückenden Schulden hinterlassen. Dass diese Generation dafür mit einem finanziell ausgezehrten Schulund Hochschulsystem groß werden muß, oder dass unter der viel zu geringen Sozialhilfe vor allem kinderreiche Familien zu leiden haben, scheint für Rot-Grün dagegen kein Problem der „Generationengerechtigkeit“ zu sein. Inwieweit sich Finanz- und Rentenpolitik tatsächlich auf die Anforderungen einer intergenerationellen Gerechtigkeit beziehen kann, diskutiert Norbert Reuter in seinem Aufsatz.

„Leistungsgerechtigkeit“, die Bindung von Einkommen an die eigene Leistung, gehört seit John Lockes berühmter Rechtfertigung von Privateigentum durch eigene Arbeit, zum grundlegenden Wertekanon der bürgerlichen (Leistungs)gesellschaft. Einkommen ohne entsprechende Arbeitsleistung ist daher stets legitimationsbedürftig - nur dass dieser Legitimationsbedarf durchaus unterschiedlich ge- 
sehen wird, je nachdem ob es sich um das Zins- oder Dividendeneinkommen eines Vermögensbesitzers oder um die Arbeitslosenhilfe eines für die kapitalistische Produktion überflüssigen Lohnarbeiters handelt. Während sich der letzte mit dem mehr oder weniger starken Verdacht auseinander setzen muß, nicht arbeiten zu wollen, wird der erste dafür beneidet, nicht arbeiten zu müssen. Die staatliche Unterstützung arbeitsloser LohnarbeiterInnen war daher stets mit dem Zwang verbunden, sich selbst um die Aufnahme von Lohnarbeit zu bemühen. Statt von „Arbeitszwang“ spricht man bei der modernen Sozialdemokratie lieber vom „aktivierenden Staat“ (so etwa im Schröder/Blair Papier). Von „Aktivierung" und „Workfare“ als arbeitsmarktpolitischen Instrumenten handelt der Beitrag von Hans Georg Zilian.

Nicht nur in den politischen, sondern auch in den philosophischen Debatten über Gerechtigkeit haben sich in den letzten Jahren die Koordinatensysteme maßgeblich verschoben. Die früher einmal heftig umstrittene liberale Entgegensetzung von Freiheit und Gleichheit ist inzwischen dabei die Diskussion in Gestalt eines unhintergehbaren Ausgangspunktes zu dominieren. Bernd Ladwig diskutiert diese gängige Entgegensetzung von Freiheit und Gleichheit und macht deutlich, dass keine Vorstellung von Ge- rechtigkeit ohne ein Minimum an Gleichheit auskommen kann. Aber schon ein Gerechtigkeitskonzept, das auf einer „gleichen Achtung“ jedes Menschen beruht, kann zu einer radikalen Infragestellung bestehender Ungleichheiten führen. Für einen Großteil der philosophischen und politologischen Debatten über Gerechtigkeit bildete John Rawls 1971 erschienene Theory of Justice einen wichtigen Bezugspunkt. Rawls hatte den Versuch unternommen, in der Tradition des liberalen vertragstheoretischen Denkens eine umfassende Theorie der Gerechtigkeit zu formulieren, deren Bezugspunkt die „geschlossene“, nationalstaatlich organisierte Gesellschaft war. Urs MüllerPlantenberg fragt nun nach den Konsequenzen von Rawls' Gerechtigkeitskonzeption unter den Bedingungen der Globalisierung.

Mit dem Problem angesichts der ökologischen Krise eine angemessene „Umweltrationalität" $\mathrm{zu}$ formulieren, setzt sich Enrique Leff auseinander. Er plädiert für eine neue „Umweltethik“, die sich allerdings nicht auf einen eng verstandenen Umweltschutz beschränken darf, sondern sich an den von der Natur abhängigen Lebensbedingungen der Menschen orientieren müsse. Mit der ökonomischen Logik des Kapitalismus, so seine These, sei eine solche Umweltethik allerdings nicht kompatibel. 